\section{Change in profile of} COVID-19 deaths in Western Cape Province, South Africa, during the fourth wave

To the Editor: Increasing evidence suggests that South Africa's fourth wave of SARS-CoV-2 infections, caused by the Omicron variant, is associated with less severe disease. ${ }^{[1-3]}$ A lower absolute number of COVID-19-related deaths has been reported in this fourth wave, and anecdotally clinicians have reported more 'incidental' COVID-19 in hospitalised patients, and fewer patients requiring oxygen support for COVID-19 pneumonia. We aimed to rapidly compare COVID-19 deaths in the third (Delta-driven) wave v. the fourth wave in Western Cape Province.

We conducted a descriptive crosssectional analysis using routine electronically available data collated by the Western Cape Provincial Health Data Centre. The first 50 deaths among patients admitted to public sector hospitals with SARS-CoV-2 infection, from when there were $5 /$ million admissions in wave 3 (15 June 2021) and wave 4 (6 December 2021), were assessed.

We included deaths during admission or within 14 days of discharge from a public sector hospital in the Western Cape. Each deceased case was independently investigated by two clinicians ( $\mathrm{HH}$ and MP) using the Single Patient Viewer ${ }^{[4]}$ by reviewing the available linked data, including laboratory tests, medication prescribed, International Statistical Classification of Diseases and Related Health Problems, 10th revision (ICD-10) coding, and electronic clinical records (emergency triage data and discharge summaries) to determine the likely cause of death. Deaths were categorised as follows:

- Severe COVID-19: evidence of COVID19 pneumonia

- No COVID-19 pneumonia:

- COVID-associated: no evidence of COVID-19 pneumonia, and presence of another medical condition as the primary cause of death, e.g. diabetic ketoacidosis, stroke, active tuberculosis or malignancies and COVID-19

- Incidental: asymptomatic COVID19 and other cause of death, where COVID-19 did not precipitate admission or death (e.g. trauma, surgery), or explicitly documented as incidental by attending clinician

- Indeterminate: insufficient information, or cannot exclude other pathology as being primary cause of death.
Differences in categorisation between the reviewers were resolved by consensus. Ethics approval was obtained from the Human Research Ethics Committee, University of Cape Town (ref. no. 460/2020).

Fifty deaths occurred within 5 days in wave 3 and within 12 days in wave 4 . In the fourth wave, $50 \%$ of COVID-19 deaths occurred in patients with evidence of COVID-19 pneumonia, compared with $78 \%$ in the third wave (Fig. 1), while $24 \%$ and $2 \%$ of COVID19 deaths were deemed to be associated with COVID in the fourth and third waves, respectively, with the most common primary diagnoses in COVID-19-associated deaths in wave 4 being malignancy (10\%) and active tuberculosis (8\%).
These findings concur with anecdotal reports from clinicians, and lower health facility bulk oxygen consumption in this fourth wave. ${ }^{[5]}$ While there is a smaller proportion of COVID-19 pneumonia in wave 4 , there are increasing numbers of COVID-associated deaths where COVID19 is not presenting with pneumonia but may have precipitated acute admission in already sick patients or may be completely incidental.

While most health authorities will continue to report 'COVID-19 deaths' as all deaths in patients with a positive COVID-19 test, these need to be interpreted appropriately in the context of increasing SARS-CoV-2 endemicity and immune

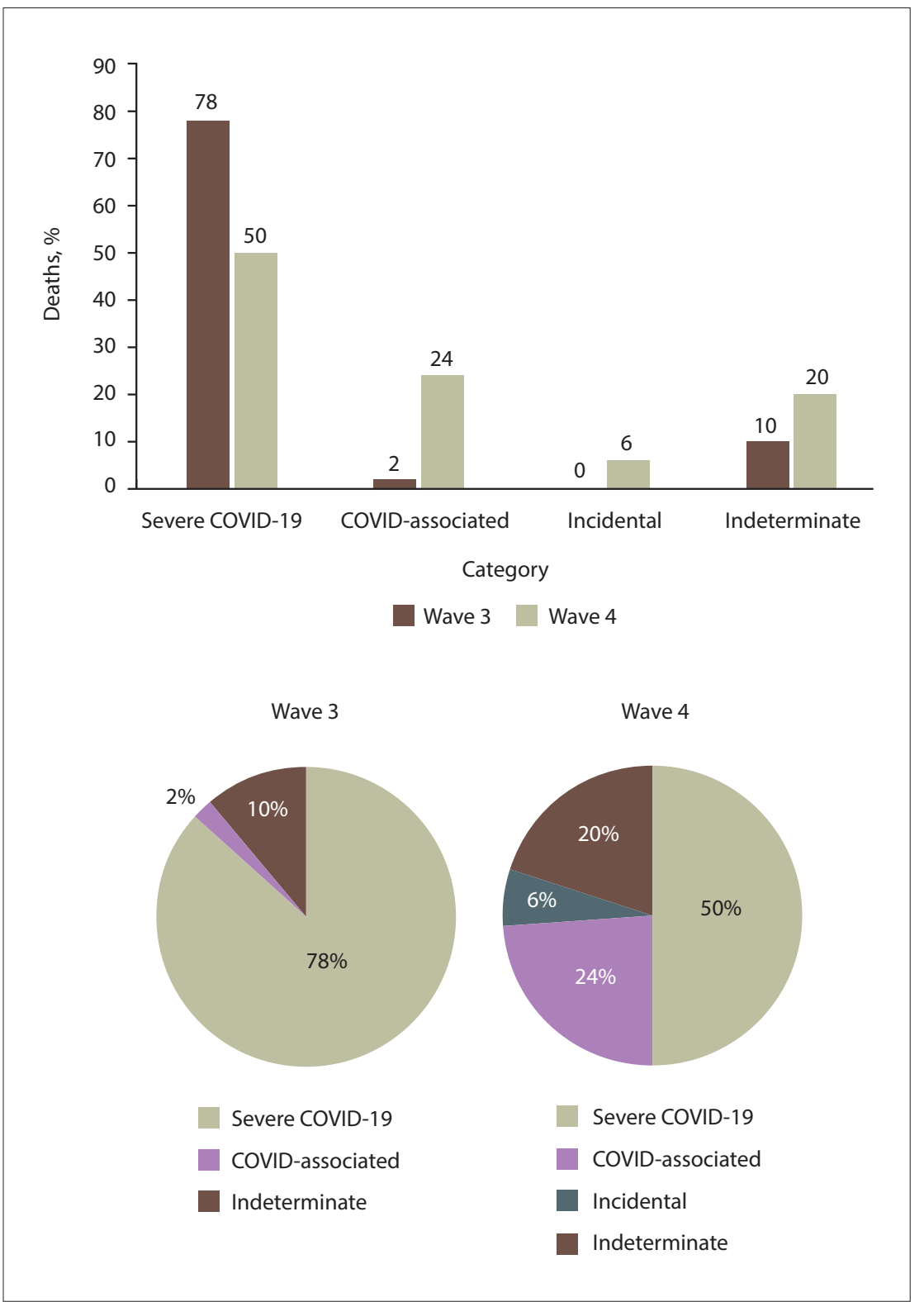

Fig. 1. Bar graph showing percentage of COVID-19 deaths in each category in wave 3 compared with wave 4 , with pie charts below showing the breakdown per wave. 
escape, widespread testing of admitted and deceased patients, and high SARS-CoV-2 prevalence at wave peaks.

\section{Masudah Paleker}

Health Intelligence, Western Cape Government Health, South Africa; Division of Health Systems and Public Health, Department of Global Health, Faculty of Medicine and Health Sciences, Stellenbosch University, South Africa

masudah.paleker@westerncape.gov.za

\section{Mary-Ann Davies}

Health Intelligence, Western Cape Government Health, South Africa; Division of Public Health Medicine, School of Public Health and Family Medicine, Faculty of Health Sciences, University of Cape Town, South Africa

\section{Peter Raubenheimer, Jonathan Naude}

Department of Medicine, Faculty of Health Sciences, University of Cape Town, South Africa

\section{Andrew Boulle, Hannah Hussey}

Health Intelligence, Western Cape Government Health, South Africa; Division of Public Health Medicine, School of Public Health and Family Medicine, Faculty of Health Sciences, University of Cape Town, South Africa

1. Wolter N, Jassat W, WalazaS, et al. Early assessment of the clinical severity of theSARS-CoV-2Omicronvariant in South Africa. medRxiv 2021 (epub 21 December 2021). https://doi.org/10.1101/2021.12.21.21268116

2. Jassat W, Abdool Karim SS, Mudara C, et al. Clinical severity of COVID-19 patients admitted to hospitals in Gauteng, South Africa during the Omicron-dominant fourth wave. Lancet 2021 (epub 29 December 2021). https://doi.org/10.2139/ssrn.3996320

3. Abdullah F, Myers J, Basu D, et al. Decreased severity of disease during the first global Omicron variant COVID-19 outbreak in a large hospital in Tshwane, South Africa. Int J Infect Dis 2021 (epub 28 December 2021). https://doi.org/10.1016/JIIIID.2021.12.357

4. Foster R, Heekes A, Hussey H, et al. Beyond interoperability with the Single Patient Viewer: A clinical portal to access integrated patient records. Int J Popul Data Sci 2020;5(5). https://doi.org/10.23889/IPPDS. V5I5.1614

5. Western Cape Government, South Africa. COVID-19 Digital Press Conference 2022 - 6 January 2022. https://coronavirus.westerncape.gov.za/weekly-digicon-presentations (accessed 12 January 2022).

S Afr Med J. Published online xx January 2022. https://doi.org/10.7196/SAMJ.2022. v112i2.16384 